\title{
Long-term effects of a placebo-controlled trial of enoxaparin for treatment of severe provoked vulvodynia
}

\author{
(1) ELAD OFIR ${ }^{1}$, (1) EILAM PALZUR², (1) JACOB BORNSTEIN ${ }^{1,2,3}$ \\ ${ }^{1}$ Azrieli Faculty of Medicine, Bar Ilan University, Safed, Israel \\ 2Research Institute, Galilee Medical Center, Nahariya, Israel \\ 32Department of Obstetrics and Gynecology, Galilee Medical Center, Nahariya, Israel
}

\section{ABSTRACT}

Objective: Provoked vulvodynia (PV) is the main cause of dyspareunia, affecting millions of women worldwide. Its cause is yet unknown, and treatment is empirical in most cases. Our purpose was to assess the long-term beneficial effects of enoxaparin on PV.

Materials and Methods: Women who previously participated in a three-month trial comparing enoxaparin to placebo for the treatment of severe PV were evaluated regarding their current pain levels using Numeric Rating Scale with various activities, whether they sought additional treatment for their condition and their satisfaction with their treatment. For pain levels, we compared time-time points within groups using a paired-sample t-test or Wilcoxon signed rank test, and we compared groups at any given time point using an independentsamples t-test or a Wilcoxon rank-sum test.

Results: Thirty-one of the 39 original participants completed the follow-up survey; 17 had been treated with enoxaparin, and 14 had received saline. Compared to their pain at the end of the prior trial, at the time of the present study, those treated with enoxaparin experienced greater decreases in pain during intercourse ( $34 \%$ decrease, $p=0.012)$ than those who received placebo $(22.5 \%$ decrease, $p=0.064)$; this was also true for other activities.

Conclusion: Enoxaparin exhibited continuing benefits three years after daily treatment for 90 days for severe PV and may have an implication for women suffering from PV.

Keywords: Enoxaparin; provoked vulvodynia; chronic pain

\section{INTRODUCTION}

Provoked vulvodynia affects millions of women worldwide. It is estimated that $3 \%-16 \%$ of women will suffer from chronic vulvar pain that will last at least 3 months. ${ }^{1}$

Patients complain that intercourse, as well as everyday activities such as sitting, tampon insertion or wearing tight clothing, become unbearable. Forty-two percent of afflicted women report significant suffering and feeling a loss of control over their lives, and they may develop secondary depression. ${ }^{1}$

Diagnosis relies on history and a physical exam and excluding conditions leading to vulvar pain. Symptoms include pain during vaginal penetration and vestibular sensitivity following localized pressure lasting at least 3 months. Eliciting pain or increased sensitivity by a cotton swab in the vestibule confirms the diagnosis. 
There are several hypotheses regarding the physiological causes of provoked vulvodynia, including genetic polymorphisms that cause an increase in pro-inflammatory factors along with a concomitant decrease in anti-inflammatory factors; ${ }^{2,3}$ decreased immune response to candida vulvovaginitis, ${ }^{4}$ inflammation of the minor vestibular glands; ${ }^{5}$ dysfunction of pelvic floor musculature; ${ }^{6}$ hormonal causes: ${ }^{7}$ bladder pain syndrome/ interstitial cystitis; ${ }^{8}$ and allergens. ${ }^{9}$ Recently, the predominant theory for provoked vulvodynia is that neuro-proliferation within the epithelial and dermis layers leads to an increase in pain sensitivity. ${ }^{10,11}$

Most treatment options are empirically based and not always successful. They include pain management, pelvic floor physical therapy and psycho-social therapy, including cognitivebehavioral therapy, as well as vestibulectomy when other measures fail.

Proliferation and degranulation of mast cells results in increased heparanase levels, ${ }^{10,11}$ causing the degradation of the extracellular matrix residing proteoglycan, heparan sulfate. This degradation leads to the release of heparin binding growth factors, enzymes and plasma proteins, thus leading to weakening of the extracellular matrix. Support for the heparanase theory comes from a previously reported study from our center (previous study) that prospectively compared patients randomly treated with subcutaneous enoxaparin, ${ }^{12}$ a heparanase inhibitor, or with saline as placebo. In that previous study, the enoxaparin-treated women showed a greater reduction in vestibular sensitivity at the end of treatment and three months later (29.6\% compared with $11.2 \%$, $\mathrm{p}=0.004$ ). Seventy-five percent (15 of 20) of them reported more than $20 \%$ pain reduction compared with $27.8 \%$ (five of 18 ) in the placebo group $(p=0.004)$. Seven enoxaparin-treated women compared with three in the placebo group had almost painless intercourse at the end of the previous study. In women who had improvement of sensitivity, a repeat biopsy at the site parallel to the original biopsy site, showed a histologically documented reduction in the number of intraepithelial-free nerve fibers in the enoxaparin treated group.

The goal of the present study was to assess the long-term effects of enoxaparin among patients suffering from provoked vulvodynia who previously participated in a 3-month trial comparing enoxaparin to placebo.

\section{MATERIAL AND METHODS}

\section{Ethical approval}

The present study was approved by the Institutional Review Board (IRB) of the Galilee Medical Center of the Israeli Health
Ministry, on June $2^{\text {nd }}$, 2013. Authorization number: 0039-13-NHR. This approval is different from the one given for the previous study. Written informed consent was obtained from all subjects.

\section{Previous study}

The present study is a follow-up to a prospective, randomized, double-blind previous study of enoxaparin treatment for provoked vulvodynia. ${ }^{12}$ Clinical trial registration of the previous study: clinicaltrials.gov, NCT00874484.

The previous study ended 3 years before the onset of the present study. ${ }^{12}$ Safety control was done using anti-factor Xa blood levels. Since enoxaparin mechanism of action involves inactivation of factor $\mathrm{Xa}$ without significant inhibition of thrombin, measurement of anti Xa activity in the previous study ascertained the prior administration of enoxaparin, and ensured that it does not exceed the therapeutic range. The previous study recruited 40 women, of which 39 were included. They were all diagnosed with provoked vulvodynia according to Friedrich's first two criteria for vulvar vestibular syndrome: severe pain in the vulvar vestibule on touch or attempted vaginal entry and tenderness to pressure localized within the vulvar vestibule. The level of provoked vulvodynia was severe, according to Marinoff's definition. ${ }^{13}$ Women were considered for enrollment to the previous study if they were aged 18-50 years, desired vaginal intercourse, had an available sexual partner, met Friedrich's first two criteria for vulvar vestibular syndrome i.e, severe pain in the vulvar vestibule on touch or attempted vaginal entry and tenderness to pressure localized within the vulvar vestibule. ${ }^{14}$ Enrollment was limited to women using an effective form of contraception, women who were postmenopausal, or had been surgically sterilized. Women were excluded if they had previous vestibulectomy, had generalized vulvodynia (constant vulvar pain, unrelated to provocation), had known hypersensitivity to heparin or enoxaparin, a positive pregnancy test, were pregnant or lactating, or planned to become pregnant during the previous study period. Women were also excluded from the previous study if they were chronic users of narcotics, had hepatic disease or clinically significant abnormal liver function tests, anticipated not being available for the entire duration of the previous study, had any coexisting significant medical condition that was likely to interfere with previous study procedures (e.g., cardiovascular, hematologic, central nervous system, pulmonary, renal). The patients were randomly and blindly assigned to self-administer either $40 \mathrm{mg}$ of enoxaparin or saline subcutaneously in the abdominal region every day for 3 months.

For the present study, patients' files were retrieved and examined. In addition, the women were contacted by telephone and asked to complete a questionnaire designed for the present 
study that examined quality of life measures using the 6 levels patient global impression of improvement (PGI-I). The pain level was assessed using the Numeric Rating Scale 0-10, frequency of intercourse per month, and treatment satisfaction. This questionnaire allowed us to compare changes over time. The first author, who made the interviews was uninvolved with the previous care of the women. He also analyzed the data with the statistician. The first author was blind of the patient treatment until the completion of the interview. In addition, the women were still blind of the previously administrated treatment at the time of the present study. Therefore, the present study is a transversal, monocentric, blinded, observational study realized on a cohort of 31 patients previously included in a randomized control trial.

\section{Present study variables}

The dependent variables were treatment satisfaction, pain level and frequency of intercourse, and the independent variable was treatment with enoxaparin.

The primary outcomes: Pain level in various aspects - pain during intercourse, pain after intercourse, finger touching the introitus, tampon insertion, riding bicycles or horses, wearing tight pants, sitting with crossed legs, urination - with no association to intercourse, urination after intercourse, and frequency of intercourse per months.

The secondary outcomes: The proportion of women who have an intimate partner, describing a high and very high satisfaction from treatment, the treatments received by the patients during the 3 years between the end of the previous study and the present study.

\section{Statistical analysis}

Data are presented as mean with standard deviation, median with the range, or proportions. In each group, changes over time were examined using paired-sample t-test or Wilcoxon signed rank test. We used an independent-sample t-test or Wilcoxon rank-sum test to compare continuous variables between groups at a given time point. We compared categorical and ordinal variables between groups using a $\chi^{2}$ test or Fisher's exact test. Based on a sample size of 14 women per group, using a pairedsample t-test with a significance of $5 \%$, we calculated that we had $88 \%$ power to detect a $20 \%$ reduction in pain levels during intercourse from the end of the previous study, which represents significant symptom improvement.

\section{RESULTS}

The present study took place from January to June 2014. Thirty one of the 39 (79\%) women from the previous study participated (Figure 1). The other eight were not located; 17 of the 31 were treated with $40 \mathrm{mg}$ enoxaparin (enoxaparin group), and 14 received saline (placebo group). Table 1 shows that the mean age of the women was 28.0 years of age in the enoxaparin group and 28.4 in the placebo group $(p=0.58)$.

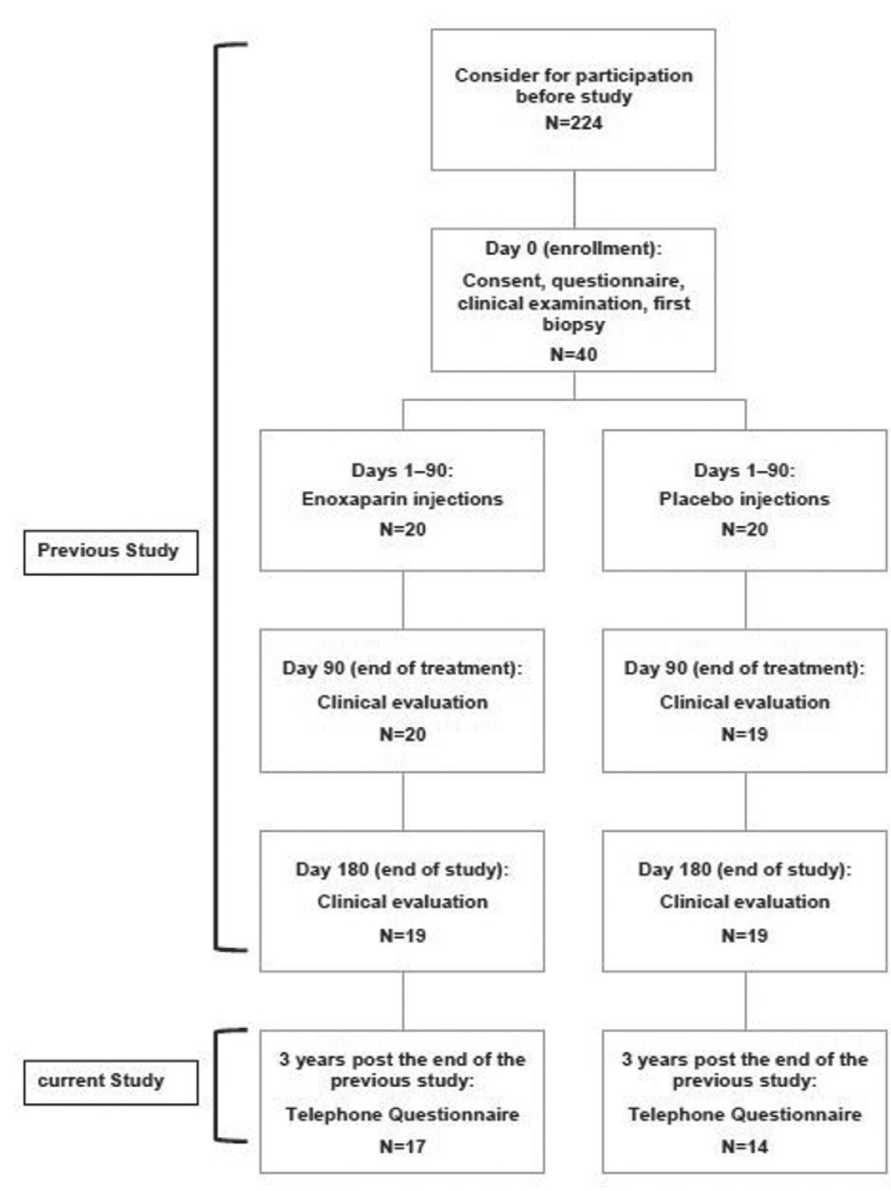

Figure 1. Flow chart of the previous and present study N: Number

\begin{tabular}{|l|l|l|l|}
\hline \multicolumn{4}{|l}{ Table 1. Patients characteristics } \\
\hline & Placebo & Enoxaparin & p-value \\
\hline Number of women & 14 & 17 & - \\
\hline Age - range & $23-36$ & $24-34$ & - \\
\hline Age - mean (SD) & $28.4(2.9)$ & $28.0(3.0)$ & $0.58+$ \\
\hline Age - median & 28.0 & 28.0 & - \\
\hline $\begin{array}{l}\text { Having intimate relationship } \\
\text { (\%) }\end{array}$ & $11(79 \%)$ & $14(82 \%)$ & $1.00 *$ \\
\hline $\begin{array}{l}\text { High and very high satisfaction } \\
\text { from treatment }\end{array}$ & $3(21.49 \%)$ & $6(35.3 \%)$ & $0.329 *$ \\
\hline $\begin{array}{l}\text { Undergone additional } \\
\text { treatment since first study (\%) }\end{array}$ & $7(50 \%)$ & $8(47.1 \%)$ & $1.00 *$ \\
\hline $\begin{array}{l}\text { Failure of study treatment } \\
\text { - reason for additional } \\
\text { treatment }\end{array}$ & $6 / 7(87.5 \%)$ & $2 / 8(25 \%)$ & $0.056++$ \\
\hline $\begin{array}{l}\text { SD: Standard deviation; +: Wilcoxon rank sum test; 2-sided; *: Fisher } \\
\text { exact test; 2-sided; ++: Chi-square test }\end{array}$ & \\
\hline
\end{tabular}


The primary outcomes: $21.5 \%$ of women treated with saline reported significant to very significant improvement compared to $35.3 \%$ of those treated with enoxaparin ( $p=0.329)$ (Table 1) Table 2 shows that when compared to the end of the previous study, women treated with enoxaparin reported a greater decrease in pain levels during intercourse than patients treated with placebo [34.5\% versus 22.5\%: the average pain level decreased from the end of the previous study until the present study, from 7.25 $(S D=2.2)$ to $4.74(S D=3.1)]$ in the enoxaparin group $(p=0.012)$, and from $7.1(\mathrm{SD}=2.6)$, to $5.45(\mathrm{SD}=2.5)$ in the placebo group $(p=0.064)$. Data shown here are paired such that baseline data only includes women who responded to the present survey.

In addition, there was a trend towards a larger decrease in pain levels among women treated with enoxaparin compared to women treated with placebo, during vestibular touch with a finger (enoxaparin: $33.3 \%$ decrease, $p=0.052$; placebo: $23.7 \%$ decrease, $p=0.081$ ); pain while riding a bicycle or horse (enoxaparin: $40 \%$ decrease, placebo: $23 \%$ increase, $p=0.2$ ), and pain with voiding (enoxaparin: $80.6 \%$ decrease; placebo: $32.5 \%$ increase in pain, $\mathrm{p}=0.785$ ) (Data not tabulated). There was no difference in pain levels between the groups for voiding after intercourse, and both groups exhibited slight increases in pain levels compared to the end of the previous study. There was no difference in intercourse frequency between the groups $(p=0.867)$. The data indicate that women treated with enoxaparin experienced a significantly greater decrease in pain levels during intercourse, as well as during other activities listed in the questionnaire, compared to women treated with placebo. Figure 2a-c show comparisons between the three different time points of the onset of the previous study, the end of the previous study, and the end of the present study, as well as an additional comparison of only the women who participated in the previous study and responded to the present study. They depict that women treated with enoxaparin reported a larger decrease in pain during intercourse and other activities such as tampon insertion, bicycle riding, and voiding after intercourse than women who received placebo.

The secondary outcome - the proportion of women who have an intimate partner, describing a high and very high satisfaction from treatment, and those who underwent additional treatments since the end of the previous study was similar in both treatment groups. The detailed results are: Eight (47.1\%) of the 17 women in the enoxaparin group and seven of the $14(50 \%)$ in the placebo group underwent additional treatments $(p=1.00)$. However, the reason the women gave for undergoing additional treatment was treatment failure in $85.7 \%$ of patients of the placebo group compared to $25 \%$ of the enoxaparin group $(p=0.056)$. The additional treatments that the women underwent were topical cream application, low oxalate diet, oral neuropathic treatments, acupuncture, physical therapy, or vestibulectomy.

\section{DISCUSSION}

The present study examined the effect of enoxaparin on women suffering from provoked vulvodynia 3 years after treatment. The main finding is that compared to women receiving placebo, treated patients experienced a decrease in pain during intercourse and in vestibular touch with a finger, in the time between the end of the previous study and the present study. Enoxaparin treatment also showed a non-significant tendency towards improvement in pain levels during other activities, including tampon insertion, bicycle or horse riding, and voiding without intercourse. Additional support for the long-lasting effectiveness of enoxaparin comes from the finding that most women in the placebo group returned for additional treatments during the 3-year period prior to the present study as compared to only one quarter of women treated with enoxaparin. These findings are supported by our sensitivity analysis restricted to

\begin{tabular}{|c|c|c|c|c|c|c|}
\hline \multirow{7}{*}{$\begin{array}{l}\text { Pain } \\
\text { during } \\
\text { intercourse }\end{array}$} & Findings & $\begin{array}{l}\text { Placebo } \\
(\mathrm{N}=14)\end{array}$ & $\begin{array}{l}\text { Enoxaparin } \\
(\mathrm{N}=17)\end{array}$ & \multicolumn{2}{|l|}{$\begin{array}{l}\text { 1-sided } \\
p \text {-value }\end{array}$} & $\begin{array}{l}\text { 2-sided } \\
\text { p-value }\end{array}$ \\
\hline & $\begin{array}{l}\text { Average pain level at end of previous } \\
\text { study (SD) }\end{array}$ & $7.1(2.6)$ & $7.25(2.2)$ & \multicolumn{2}{|l|}{-} & - \\
\hline & $\begin{array}{l}\text { Average pain level at end of current } \\
\text { study (SD) }\end{array}$ & $5.45(2.5)$ & $4.74(3.1)$ & \multicolumn{2}{|l|}{-} & - \\
\hline & Average decrease in pain (SD) & $1.6(2.7)$ & $2.5(3.7)$ & \multicolumn{2}{|l|}{-} & $* 0.427$ \\
\hline & $\begin{array}{l}\% \text { decrease in pain from end of } \\
\text { previous study to end of current study }\end{array}$ & $22.5 \%$ & $34.5 \%$ & $\begin{array}{l}\text { Placebo: } \\
* 0.064\end{array}$ & $\begin{array}{l}\text { Enoxaparin: } \\
* 0.012\end{array}$ & - \\
\hline & $\%$ women with a decrease $>20 \%$ & $45.5 \%$ & $62.5 \%$ & \multicolumn{2}{|l|}{-} & ${ }^{++} 0.452$ \\
\hline & $\%$ women with a decrease $>30 \%$ & $36.4 \%$ & $43.8 \%$ & \multicolumn{2}{|l|}{-} & ${ }^{++} 1.00$ \\
\hline
\end{tabular}



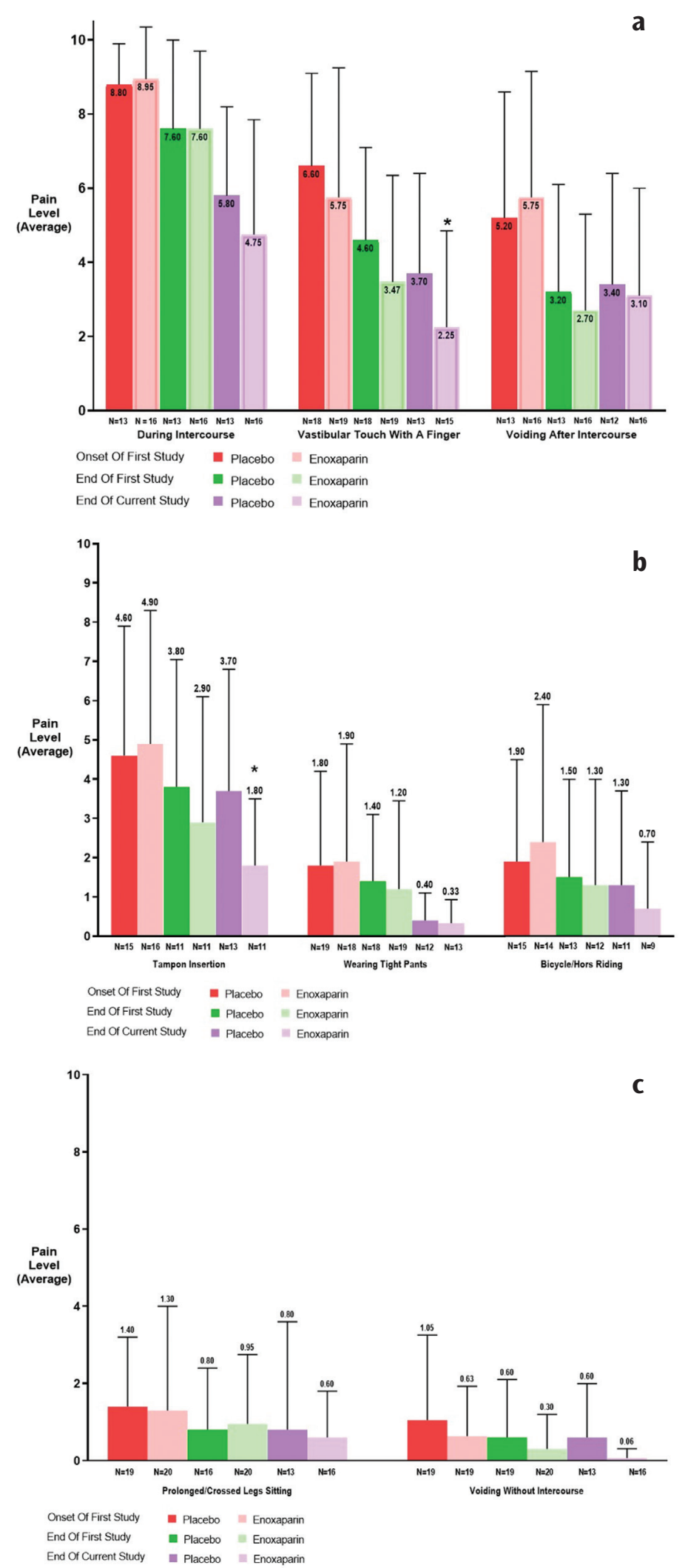

Figure 2a-c. Comparison of pain levels of women suffering from provoked vulvodynia during intercourse and other activities at three time points: onset of previous study, end of previous study, end of present study. Legend to Figure $2 a: * p \leq 0.05 ; \mathrm{N}$ : Number only women who answered the same questions in both studies. The fact that pain levels in the treatment group unexpectedly continued to decrease up until the end of the present study, three years after treatment completion, suggests that not only is enoxaparin effective at treating provoked vulvodynia, but that it may promote healing as well, or, that by blocking heparanase, new neuroproliferation into the epithelium subsided, so that allodynia gradually diminished. The present study is the first to examine the long-term efficacy of enoxaparin as a treatment for provoked vulvodynia.

One of the parameters we tested as a marker for successful longterm treatment is the reason why patients sought additional treatments. While a similar proportion of women in both groups sought additional treatments, over three-quarters of the placebo group were referred due to a complete lack of symptom alleviation, as compared to only one quarter of the patients treated with enoxaparin that received additional treatment for mild residual discomfort. This suggests that, even 3 years after completion of treatment, enoxaparin continues to improve quality of life and sexual function.

The physiological basis for the efficacy of enoxaparin for treatment of provoked vulvodynia originated from the observation of proliferation and infiltration of neurons into the epithelium of affected patients, which leads to increased pain sensitivity. ${ }^{10-12}$ These findings, together with an observed increase of mast cells, raised the hypothesis that heparanase secretion from mast cells allows the infiltration of neurons into the epithelium and the stroma. ${ }^{10,11}$ The previous study tested whether blocking heparanase activity with enoxaparin would alleviate pain. Indeed, the previous study found significant symptom improvement following a short-term treatment of 3 months. ${ }^{12}$ The present study examined the long-term effects of this treatment and found that the positive effect of enoxaparin is sustained for at least 3 years. This result strengthens the idea that blocking heparanase treats the etiology of provoked vulvodynia. As a next step, it will be helpful to examine similar, yet more specific, heparanase blockers. In order to examine whether enoxaparin affects the tissue itself over the long term, biopsies of women treated with enoxaparin should be obtained 3 years after treatment and be histologically examined for free nerve fiber endings and mast cell number. This can then be compared to biopsies taken at the end of the previous study.

In order to assess treatment efficacy, enoxaparin must be compared to other treatments for provoked vulvodynia. Treatment of provoked vulvodynia usually begins with medications, either locally or orally administered. One pharmaceutical option is Amitriptyline, yet most studies have 
been unable to show any significant increase in quality of life or decrease in pain levels following treatment. ${ }^{15}$ In addition, side effects of Amitriptyline treatment, including trouble concentrating, tachycardia, hypotension, seizures, constipation, dry mouth and urinary retention, present significant drawbacks. Surgical vulvar vestibulectomy is currently the most effective treatment and will usually be recommended after failure of less invasive measures. One study demonstrated a significant decrease of pain during intercourse and an increase in sexual activity in $90 \%$ of surgically treated patients. ${ }^{16}$ However, some patients report worsening of their condition, including pain reoccurrence, vaginal dryness and Bartholin's duct occlusion, which are conditions requiring additional surgery. ${ }^{17}$ The findings of the present study place enoxaparin as a possible effective alternative to surgical intervention.

One less encouraging result of the present study is the fact that treatment was effective in only a portion of patients. This suggests that provoked vulvodynia may be a multi-causal disease and that enoxaparin is effective only in a subset of patients.

The present study's main advantage is the long-term evaluation of a novel treatment of provoked vulvodynia and comparing two groups that were originally randomized to treatment and control groups. One drawback of the present study is that the average age of the participants was young, with mean age 28 (enoxaparin group) to 28.4 (placebo group) years old, and they all suffered from severe provoked vulvodynia. Therefore, results may be different in older patients and/or in patients suffering from a less severe form of the disease.

Another advantage is that the interviews were made by a researcher who was uninvolved with the primary care of the women. This prevented a bias that might have been introduced if women wanted not to offer disappointing answers to the primary researcher who followed them during the previous study.

A limitation of the present study is that it is a relatively small study, the evaluation was made by using questionnaires only, and no new biopsies were made. During the three years, women had different interventions to try to control the pain. Not all patients who participated in the previous study were located. However, $79 \%$ were found. This significant rate assures that the findings of the present study are highly reliable.

\section{CONCLUSION}

The present study shows that women with severe provoked vulvodynia who had previously participated in a three-month trial comparing enoxaparin to placebo for the treatment of provoked vulvodynia were contacted three years later with self- questionnaire reported less pain with intercourse if they were in the enoxaparin group. If other studies substantiate this finding, enoxaparin may have an implication for women suffering from provoked vulvodynia.

\section{Contributions}

Surgical and/or Medical Practices: E.O., E.P., J.B., Concept: E.O., E.P., J.B., Design: E.O., E.P., J.B., Data Collection or Processing: E.O., E.P., J.B., Analysis or Interpretation: E.O., E.P., J.B., Literature Search: E.O., E.P., J.B., Writing: E.O., E.P., J.B.

\section{Ethics}

Ethics Committee Approval: The present study was approved by the Institutional Review Board (IRB) of the Galilee Medical Center of the Israeli Health Ministry, on June $2^{\text {nd }}, 2013$. Authorization number: 0039-13-NHR.

Informed Consent: Written informed consent was obtained from all subjects.

Peer-review: Externally peer-reviewed.

\section{DISCLOSURES}

Conflict of Interest: No conflict of interest was declared by the authors.

Financial Disclosure: The authors declared that this study received no financial support.

\section{REFERENCES}

1. Bergeron S, Reed BD, Wesselmann U, Bohm-Starke N. Vulvodynia. Nat Rev Dis Primers 2020; 6: 36.

2. Gerber S, Bongiovanni AM, Ledger WJ, Witkin SS. Interleukin-1beta gene polymorphism in women with vulvar vestibulitis syndrome. Eur J Obstet Gynecol Reprod Biol 2003; 107: 74-7.

3. Jeremias J, Ledger WJ, Witkin SS. Interleukin 1 receptor antagonist gene polymorphism in women with vulvar vestibulitis. Am J Obstet Gynecol 2000; 182: 283-5.

4. Pyka RE, Wilkinson EJ, Friedrich EG Jr, Croker BP. The histopathology of vulvar vestibulitis syndrome. Int J Gynecol Pathol 1988; 7: 249-57.

5. Foster DC, Piekarz KH, Murant TI, LaPoint R, Haidaris CG, Phipps RP. Enhanced synthesis of proinflammatory cytokines by vulvar vestibular fibroblasts: implications for vulvar vestibulitis. Am J Obstet Gynecol 2007; 196: 346.e1-8.

6. Glazer HI, Rodke G, Swencionis C, Hertz R, Young AW. Treatment of vulvar vestibulitis syndrome with electromyographic biofeed back of pelvic floor musculature. J Reprod Med 1995; 40: 283-90.

7. Harlow BL, Vitonis AF, Stewart EG. Influence of oral contraceptive use on the risk of adult-onset vulvodynia. J Reprod Med 2008; 53 : 102-10. 
8. Tarr G, Selo-Ojeme DO, Onwude JL. Coexistence of vulvar vestibulitis and interstitial cystitis. Acta Obstet Gynecol Scand 2003; 82: 969.

9. Babula O, Bongiovanni AM, Ledger WJ, Witkin SS. Immunoglobulin E antibodies to seminal fluid in women with vulvar vestibulitis syndrome: relation to onset and timing of symptoms. Am J Obstet Gynecol 2004; 190: 663-7.

10. Bornstein J, Goldschmid N, Sabo E. Hyperinnervation and mast cell activation may be used as histopathologic diagnostic criteria for vulvar vestibulitis. Gynecol Obstet Invest 2004; 58: 171-8.

11. Bornstein J, Cohen Y, Zarfati D, Sela S, Ophir E. Involvement of heparanase in the pathogenesis of localized vulvodynia. Int J Gynecol Pathol 2008; 27: 136-41.

12. Farajun Y, Zarfati D, Abramov L, Livoff A, Bornstein J. Enoxaparin treatment for vulvodynia: a randomized controlled trial. Obstet Gynecol 2012; 120: 565-72.
13. Marinoff SC, Turner ML. Vulvar vestibulitis syndrome: an overview. Am J Obstet Gynecol 1991; 165: 1228-33.

14. Friedrich EG Jr. Vulvar vestibulitis syndrome. J Reprod Med 1987; 32: $110-4$.

15. Schmidt S, Bauer A, Greif C, Merker A, Elsner P, Strauss B. Vulvar pain. Psychological profiles and treatment responses. J Reprod Med 2001; 46: 377-84.

16. Gaunt G, Good A, Stanhope CR. Vestibulectomy for vulvar vestibulitis. J Reprod Med 2003; 48: 591-5.

17. Schneider D, Yaron M, Bukovsky I, Soffer Y, Halperin R. Outcome of surgical treatment for superficial dyspareunia from vulvar vestibulitis. J Reprod Med 2001; 46: 227-31. 